\title{
INTRODUCTION
}

\section{Guidelines for colorectal cancer screening in high risk groups}

\section{Edited by S Cairns, J H Scholefield}

The British Society of Gastroenterology (BSG) and the Association of Coloproctolgy for Great Britain and Ireland (ACPGBI) commissioned these guidelines. The aim is to provide guidance on the appropriateness, method, and frequency of screening people with diseases known to be associated with colorectal cancer. There is increasing awareness among relatives of patients with colorectal cancer that they may be at risk from this disease and there is rising demand for screening. These guidelines are for members of gastroenterology teams, primary care physicians, purchasers of health care, and people thought to be at increased risk of colorectal cancer.

See end of article for authors' affiliations

Correspondence to: Dr S Cairns, Digestive Diseases Centre, Royal Sussex County Hospital Eastern Road, Brighton BN2 5BE, UK:

Stuart.Cairns@bsuh.nhs.uk
C olorectal cancer is the second most common cause of cancer related death and the third most common cancer in the UK, affecting more than 30000 people each year with an average five year survival rate of $40 \% .^{1-4}$ Currently, $80 \%$ of cases are not diagnosed until the cancer has spread through the bowel wall or beyond. ${ }^{5}$ Such cases have a much worse prognosis than cancers confined to the bowel wall. As many patients with colorectal cancer do not develop symptoms until the cancer is advanced, the detection of a greater proportion of cases at an earlier stage can only be achieved by the screening of asymptomatic persons. Controversy remains about the appropriateness of and preferred methods for screening a population but there is more consensus about the need for screening people known to be at increased risk. People with family histories giving a lifetime risk of less than 1 in 10 , do not generally qualify for screening given the resources currently available.

It is recognised that inquiry into a family history of colorectal cancer may provide inaccurate information for a variety of reasons, for example no contact with family members or inaccurate information about family illness. Guidelines on genetic screening given within this document will inevitably become outdated, hence we have included a review date. Similarly, with emerging technological developments such as virtual colonoscopy the guidelines will require review.

PROCESS OF GUIDELINE FORMULATION

Experts within the fields of medical and surgical gastroenterology and clinical genetics were requested to submit guidelines in a defined format
(The BSG Framework for guidelines in Gastroenterology) for the various high risk groups. Information in these guidelines has only been included if it was published in a peer reviewed article. The evidence base arose from sources including the Cochrane Library, Medline and Embase. Two reviewers (SC and JS) suggested amendment as necessary to comply with the format and ensure consistency. The Clinical Services and Standards Committee of the BSG and ACGBI then reviewed and modified the guidelines.

The review date for this guideline is 2006 .

The guidelines were produced to conform to the system proposed by the North of England evidence based guidelines development project. ${ }^{78}$

\section{VALIDITY AND GRADING OF RECOMMENDATIONS Categories of evidence}

Ia: Evidence obtained from meta-analysis of randomised controlled trials.

Ib: Evidence obtained from at least one randomised controlled trial.

IIa: Evidence obtained from at least one well designed controlled study without randomisation.

IIb: Evidence obtained from at least one other type of well designed quasi-experimental study.

III: Evidence obtained from a well designed nonexperimental descriptive study, such as comparative studies, correlation studies, and case studies. IV: Evidence obtained from expert committee reports or opinions or clinical experiences of respected authorities.

The evidence category is indicated in parentheses within the reference section.

\section{Grading of recommendations}

The strength of each recommendation is dependent upon the category of the evidence supporting it, and is graded according to the following system.

A: Evidence categories Ia and Ib.

B: Evidence categories IIa, IIb, III

C: Evidence category IV.

\section{GAINING INFORMED CONSENT FOR SCREENING}

The public has misconceptions about the purpose and accuracy of screening and surveillance." These misconceptions are particularly relevant to screening for bowel cancer because of the invasive nature of the investigations needed. Therefore patients should be aware that surveillance cannot 
guarantee a reduced cancer risk but rather offers a reasonable chance of risk reduction..$^{10}$ Detrimental effects of screening may include one or more of the following: anxiety, false alarms, false reassurance, overdiagnosis, and overtreatment. The particular risks of colorectal screening are the complications of colonoscopy and polypectomy, which include colonic perforation, haemorrhage, and death. It is crucial that patients undergoing screening are fully aware of both the benefits and harm, particularly when screening entails procedures with recognised risks. As this combination of benefit and harm affects any person offered screening, each must receive full and accurate information about the estimate of their individual risk of developing colorectal cancer as well the risks of screening.

....................

Authors' affiliations

S Cairns, Digestive Diseases Centre, Royal Sussex County Hospital, Brighton, UK

J H Scholefield, Department of Surgery, University Hospital,

Nottingham, UK

\section{REFERENCES}

1 Office for National Statistics. Health Statistics Quarterly 6. London: ONS, Summer 2000.

2 Scottish Health Statistics 1999. Edinburgh: Scottish Cancer Intelligence Unit, ISD Scotland, 1999.

3 General Register Office, Scotland. 1998 Annual report. Edinburgh: General Register Office, 1998.

4 Northern Ireland Cancer Registry. Cancer incidence data 1993-6 and mortality data 1993-8. Belfast: Northern Ireland Cancer Registry 1999.

5 Allum WH, Slaney G, McCOnkey CC, et al. Cancer of the colon and rectum in the West Midlands 1957-1981. Br J Surg 1994:81:1060-3.

6 McArdle CS, Hole D, Hansell D, et al. Prospective study of colorectal cancer in the West of Scotland: ten year follow up. Br J Surg 1990;70:280-2.

7 Eccles M, Clapp Z, Grimshaw J, et al. North of England evidence based guidelines development project: methods of guidelines development. BM 1996;312:760-2.

8 Grimshaw J, Eccles M, Russell I. Developing clinically valid practice guidelines. Journal of Evaluation in Clinical Practice 1995; 1:37-48.

9 Woloshin S, Schwartz LM, Byram SJ, et al. Women's understanding of the mammographic screening debate. Ann Intern Med 2000;160: 1434-10.

10 Lennard-Jones JE, Young GP, Rozen P, et al, eds. Prevention and early detection of colorectal cancer. London: W B Saunders, 1996:217-38. 